\title{
MEASUREMENT OF BORON AND CARBON FLUXES IN COSMIC RAYS WITH THE PAMELA EXPERIMENT
}

\author{
O. Adriani ${ }^{1,2}$, G. C. Barbarino ${ }^{3,4}$, G. A. Bazilevskaya ${ }^{5}$, R. Bellotti ${ }^{6,7}$, M. Boezio ${ }^{8}$, E. A. Bogomolov ${ }^{9}$, M. Bongi $^{1,2}$,

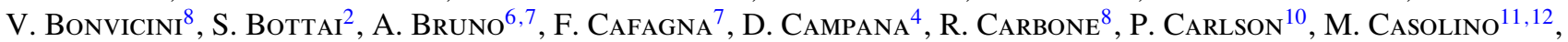 \\ G. Castellini ${ }^{13}$, I. A. Danilchenko ${ }^{14}$, C. De Donato ${ }^{11,15}$, C. De Santis ${ }^{11,15}$, N. De Simone ${ }^{11}$, V. Di Felice ${ }^{11,16}$, \\ V. Formato ${ }^{8,17}$, A. M. Galper ${ }^{14}$, A. V. Karelin ${ }^{14}$, S. V. Koldashov ${ }^{14}$, S. Koldobskiy ${ }^{14}$, S. Y. Krutkov ${ }^{9}$, A. N. Kvashnin ${ }^{5}$, \\ A. Leonov $^{14}$, V. MalaKhov ${ }^{14}$, L. Marcelli ${ }^{11,15}$, M. Martucci ${ }^{15}, 18$, A. G. Mayorov ${ }^{14}$, W. MenN ${ }^{19}$, M. Mergé $^{11,15}$, \\ V. V. Mikhailov ${ }^{14}$, E. Mocchiutti ${ }^{8}$, A. Monaco ${ }^{6,7}$, N. Mori $^{2,20}$, R. Munini ${ }^{8,17}$, G. Osteria ${ }^{4}$, F. Palma ${ }^{11,15}$, B. Panico ${ }^{4}$,

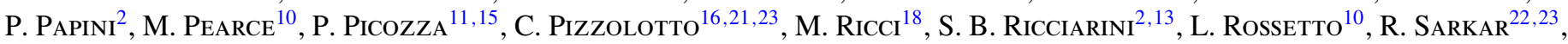

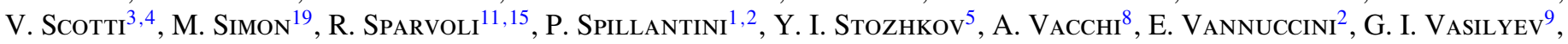

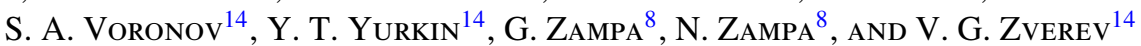 \\ ${ }^{1}$ Department of Physics and Astronomy, University of Florence, I-50019 Sesto Fiorentino, Florence, Italy \\ 2 INFN, Sezione di Florence, I-50019 Sesto Fiorentino, Florence, Italy \\ ${ }^{3}$ Department of Physics, University of Naples "Federico II," I-80126 Naples, Italy \\ ${ }^{4}$ INFN, Sezione di Naples, I-80126 Naples, Italy \\ ${ }^{5}$ Lebedev Physical Institute, RU-119991 Moscow, Russia \\ ${ }^{6}$ Department of Physics, University of Bari, I-70126 Bari, Italy \\ ${ }^{7}$ INFN, Sezione di Bari, I-70126 Bari, Italy \\ 8 INFN, Sezione di Trieste, I-34149 Trieste, Italy \\ ${ }^{9}$ Ioffe Physical Technical Institute, RU-194021 St. Petersburg, Russia \\ ${ }^{10}$ KTH, Department of Physics, and the Oskar Klein Centre for Cosmoparticle Physics, AlbaNova University Centre, SE-10691 Stockholm, Sweden \\ ${ }^{11}$ INFN, Sezione di Rome "Tor Vergata," I-00133 Rome, Italy \\ ${ }^{12}$ RIKEN, Advanced Science Institute, Wako-shi, Saitama, Japan \\ 13 IFAC, I-50019 Sesto Fiorentino, Florence, Italy \\ ${ }^{14}$ National Research Nuclear University MEPhI, RU-115409 Moscow, Russia \\ 15 Department of Physics, University of Rome "Tor Vergata," I-00133 Rome, Italy \\ ${ }^{16}$ Agenzia Spaziale Italiana (ASI) Science Data Center, Via del Politecnico snc, I-00133 Rome, Italy \\ ${ }^{17}$ Department of Physics, University of Trieste, I-34147 Trieste, Italy \\ ${ }^{18}$ INFN, Laboratori Nazionali di Frascati, Via Enrico Fermi 40, I-00044 Frascati, Italy \\ ${ }^{19}$ Department of Physics, Universität Siegen, D-57068 Siegen, Germany \\ ${ }^{20}$ Centro Siciliano di Fisica Nucleare e Struttura della Materia (CSFNSM), Viale A. Doria 6, I-95125 Catania, Italy \\ ${ }^{21}$ INFN, Sezione di Perugia, I-06123 Perugia, Italy \\ 22 Indian Centre for Space Physics, 43 Chalantika, Garia Station Road, Kolkata 700084, West Bengal, India \\ Received 2014 April 16; accepted 2014 July 2; published 2014 July 31
}

\begin{abstract}
The propagation of cosmic rays inside our galaxy plays a fundamental role in shaping their injection spectra into those observed at Earth. One of the best tools to investigate this issue is the ratio of fluxes for secondary and primary species. The boron-to-carbon $(\mathrm{B} / \mathrm{C})$ ratio, in particular, is a sensitive probe to investigate propagation mechanisms. This paper presents new measurements of the absolute fluxes of boron and carbon nuclei as well as the B/C ratio from the PAMELA space experiment. The results span the range $0.44-129 \mathrm{GeV} / \mathrm{n}$ in kinetic energy for data taken in the period 2006 July to 2008 March.
\end{abstract}

Key words: astroparticle physics - cosmic rays

Online-only material: color figures

\section{INTRODUCTION}

Propagation in the interstellar medium (ISM) significantly affects the spectrum of galactic cosmic rays. After being accelerated by high-energy astrophysical processes such as supernovae explosions, cosmic rays are injected into the interstellar space, propagate through it and eventually reach the Earth where they are detected. The multitude of physical processes that cosmic rays undergo during propagation (e.g., diffusion, spallation, emission of synchrotron radiation, etc.) shape the injection spectra and chemical composition into the observed values. A detailed knowledge of these processes is therefore needed in order to interpret the experimental data in terms of source parameters, or in estimating the expected background when searching for contributions from new sources.

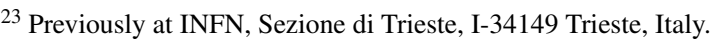

There is still a relatively high degree of uncertainty regarding the physical processes relevant to propagation of cosmic rays and the impact of experimental uncertainties on the determination of propagation parameters (see Maurin et al. 2010 and references therein). The propagation is usually modeled in terms of a diffusive transport equation (Ptuskin 2012). The equation contains terms that account for diffusion in the irregular galactic magnetic field, convection due to the galactic wind, energy losses, re-acceleration (modeled as diffusion in momentum space), spallation and radioactive decay, and source terms. Some parameters of the equation are simply related to directly measurable quantities unrelated to cosmic rays, and thus they can be obtained from independent measurements (e.g., the density of atomic hydrogen in the ISM, which is needed in order to estimate the spallation rate, can be measured by means of $21 \mathrm{~cm}$ radio surveys). Other parameters are obtained by fitting distributions derived from numerical propagation models like GALPROP (Strong \& Moskalenko 1998; Vladimirov 
2012) or DRAGON (Gaggero et al. 2013) to direct cosmic ray measurements.

In order to test and tune the propagation models, a particularly useful measurable quantity is the secondary to primary flux ratio. Primary nuclei are those accelerated by cosmic ray sources such as supernova remnants, whereas secondaries are those produced in interactions of primaries with the ISM during propagation. The boron to carbon flux ratio $(\mathrm{B} / \mathrm{C})$ has been widely studied. Since boron is produced in negligible quantities by stellar nucleosynthesis processes (Bethe 1939), almost all of the observed boron is believed to be from spallation reactions of $\mathrm{CNO}$ primaries on atomic and molecular $\mathrm{H}$ and $\mathrm{He}$ present in the ISM. The $\mathrm{B} / \mathrm{C}$ flux ratio is therefore a clean and direct probe of propagation mechanisms, and it is considered as the "standard tool" for studying propagation models (Strong et al. 2007; Obermeier et al. 2012).

The $\mathrm{B} / \mathrm{C}$ flux ratio, as well as the absolute boron and carbon fluxes, have been measured by balloon-borne (Freier et al. 1959; Panov et al. 2008; Ahn et al. 2008; Obermeier et al. 2011) and by space-based experiments (Engelmann et al. 1990; Swordy et al. 1990; Webber et al. 2002; Aguilar et al. 2010; Lave et al. 2013; Oliva et al. 2013), with different techniques and spanning various energy ranges from about $80 \mathrm{MeV} / \mathrm{n}$ up to a few $\mathrm{TeV} / \mathrm{n}$. Even if the spread in the measurements and their associated errors makes it difficult to clearly discriminate between the various models or to tightly constrain model parameters, there is a general consensus about several points. The relative abundance of the light elements $\mathrm{Li}, \mathrm{Be}$, and $\mathrm{B}$ in cosmic rays is significantly higher than in the solar system (de Nolfo et al. 2006). This supports the idea of creation by spallation reactions in ISM. The $\mathrm{B} / \mathrm{C}$ flux ratio has a peak value at $\sim 1 \mathrm{GeV} / \mathrm{n}$, which can favor a model with distributed stochastic re-acceleration (Letaw et al. 1993). The B/C flux ratio decreases at high energies and its shape, in diffusive models, is mainly determined by the energy dependence of the diffusion coefficient (Castellina \& Donato 2005).

In this paper, a new set of measurements of boron and carbon fluxes as well as the $\mathrm{B} / \mathrm{C}$ flux ratio obtained with the PAMELA instrument in the kinetic energy range $0.44-129 \mathrm{GeV} / \mathrm{n}$ during the solar minimum period spanning from 2006 July to 2008 March are presented. The study of solar modulation effects on the low-energy component of the spectra over a longer time interval will be the subject of a future publication. After a brief description of the PAMELA detector system, the analysis techniques and an evaluation of systematic uncertainties are presented, followed by a discussion of the results.

\section{THE PAMELA DETECTOR}

A schematic view of the PAMELA detector system (Picozza et al. 2007) is shown in Figure 1. The design was chosen to meet the main scientific goal of precisely measuring the light components of the cosmic ray spectrum in the energy range starting from tens of $\mathrm{MeV}$ up to $1 \mathrm{TeV}$ (depending on particle species), with a particular focus on antimatter. To this end, the design is optimized for $|Z|=1$ particles and to provide a high lepton-hadron discrimination power. The core of the instrument is a magnetic spectrometer (Adriani et al. 2007) made by six double-sided silicon microstrip tracking layers placed in the bore of a permanent magnet. The read-out pitch of the silicon sensors is $51 \mu \mathrm{m}$ in the $\mathrm{X}$ (bending) view and $66.5 \mu \mathrm{m}$ in the $\mathrm{Y}$ view. The spectrometer provides information about the magnetic rigidity $\rho=p c /(Z e)$ of the particle (where $p$ and $Z$ are the particle momentum and the electric charge, respectively).

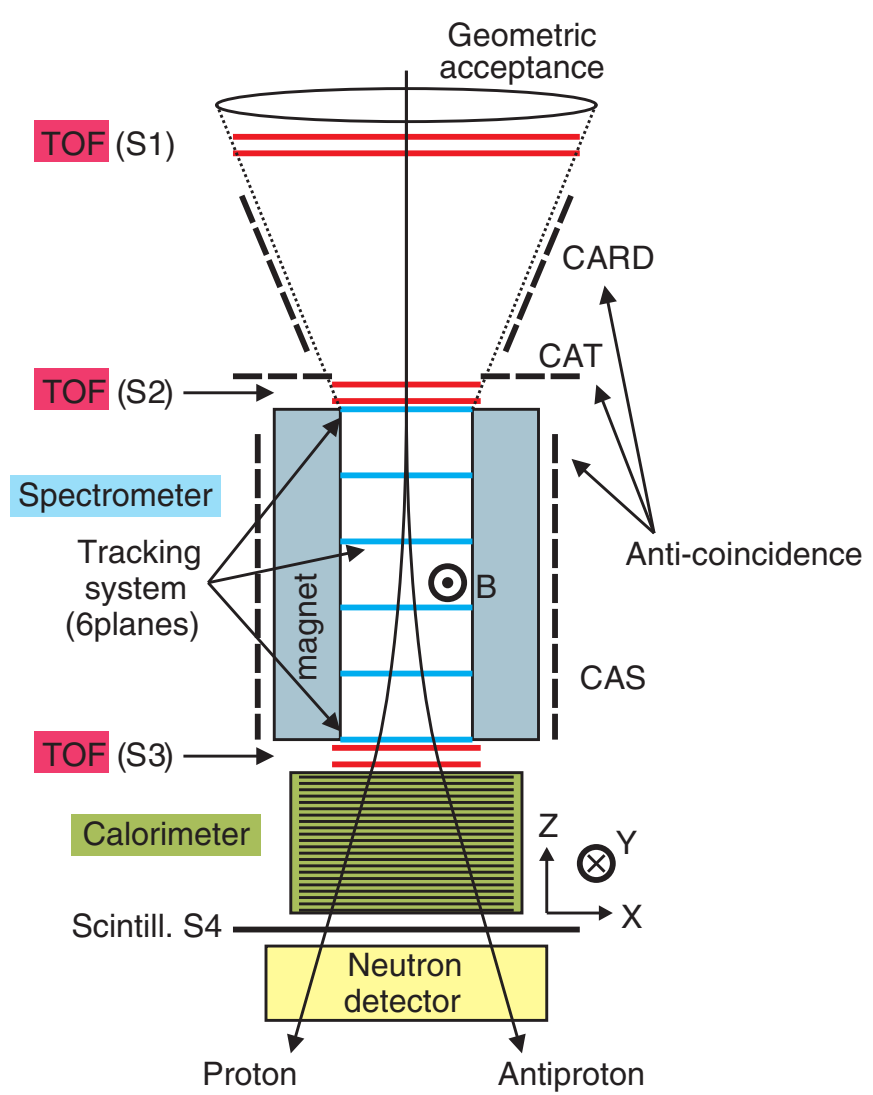

Figure 1. Schematic view of the PAMELA apparatus.

(A color version of this figure is available in the online journal.)

Six layers of plastic scintillator paddles arranged in three $X-Y$ planes (S1, S2, and S3 in Figure 1) placed above and below the magnetic cavity constitute the time-of-flight (TOF) system (Barbarino et al. 2008; Osteria \& Russo 2008). The flight time of particles is measured with a time resolution of $250 \mathrm{ps}$ for $|Z|=1$ particles and about $70 \mathrm{ps}$ for boron and carbon nuclei (Campana et al. 2009). This allows albedo particles to be rejected and, in combination with the track length information obtained from the tracking system, precise measurement of the particle velocity, $\beta=v / c$. The TOF scintillators can identify the absolute particle charge up to oxygen by means of six independent ionization measurements. The tracking system and the upper TOF system are shielded by an anticoincidence system (AC; Pearce et al. 2003) made of plastic scintillators and arranged in three sections (CARD, CAT, and CAS in Figure 1), which allows spurious triggers generated by secondary particles to be rejected during offline data analysis. A sampling electromagnetic calorimeter (Boezio et al. 2002; Bonvicini et al. 2009) is placed below S3. It consists of 22 modules, each comprising a tungsten converter layer placed between two layers equipped with single-sided silicon strip detectors with orthogonal read-out strips. The total depth of the calorimeter is $16.3 X_{0}$, while the readout pitch of the strips is $2.44 \mathrm{~mm}$. The calorimeter measures the energy of electrons and positrons, and gives a lepton/hadron rejection power of $\sim 10^{5}$ by means of topological shower analysis, thanks to its fine lateral and longitudinal segmentation. A tail-catcher scintillating detector (S4) and a neutron detector placed below the calorimeter help to further improve the rejection power.

The geometric factor of the apparatus, defined by the magnetic cavity, is energy dependent because of the track curvature induced by the magnetic field, and increases as the energy of the 
particle increases. However, for rigidities above $1 \mathrm{GV}$ it varies only by a few per mil, reaching the value of $21.6 \mathrm{~cm}^{2} \mathrm{sr}$ at the highest rigidity.

The PAMELA apparatus was launched on 2006 June 15, and has been continuously taking data since then. It is hosted as a piggyback payload on the Russian satellite Resurs-DK1, which executes a $70^{\circ}$ semi-polar orbit. The orbit was elliptical with variable height between 350 and $620 \mathrm{~km}$ up to 2010, after which it was converted to the current circular orbit with height about $600 \mathrm{~km}$.

\section{DATA ANALYSIS}

\subsection{Data Processing}

The event reconstruction routines require silicon strips to be gathered into clusters. A "seed" strip is defined as a strip with a signal-to-noise ratio $(\mathrm{S} / \mathrm{N})$ greater than 7; it is grouped with its neighboring "signal" strips with $\mathrm{S} / \mathrm{N}>4$ to form a cluster. For each cluster an estimate of the particle impact point is obtained by means of an analog position finding algorithm (Adriani et al. 2007). The original reconstruction routines were conceived and tuned to deal with $|Z| \sim 1$ particles. However the higher ionization energy losses of boron and carbon in the silicon layers of the tracking system saturate the front-end electronics, leading to a degradation of the performance of position finding with respect to $|Z| \sim 1$ particles. A different position finding algorithm has thus been implemented for this saturation regime. For each cluster of silicon strips, the saturated strips have been treated as if read-out system was digital, and the impact point has been evaluated as the geometric center. The associated spatial resolution can be approximated as the readout pitch over $\sqrt{12}$, which translates to $\sim 14 \mu \mathrm{m}$ for the $\mathrm{X}$ (bending) view and $\sim 19 \mu \mathrm{m}$ for the $\mathrm{Y}$ view. The associated maximum detectable rigidity $\left(\mathrm{MDR}^{24}\right)$ is $\sim 250 \mathrm{GV}$.

Prior to event reconstruction, the clusters with an associated energy release less than $5 \mathrm{MIP}^{25}$ have been removed. This helps to eliminate clusters associated with delta rays and light secondary particles, e.g., backscattered particles from the calorimeter. There is a twofold effect: the tracking efficiency is increased since the tracking algorithm has less clusters to deal with, and the energy dependence of the tracking efficiency is reduced at high energies by removing backscattering clusters, which are mainly produced by high-energy primaries interacting in the calorimeter.

\subsection{Event Selection}

In order to be able to reliably measure the magnetic rigidity, events with a single track in the spectrometer containing at least four hits in the $\mathrm{X}$ view and three hits in the $\mathrm{Y}$ view have been selected. A good $\chi^{2}$ value for the fitted track was required. The $\chi^{2}$ distribution is energy dependent and thus the selection criterion has been calibrated in order to obtain a constant efficiency of about $90 \%$ over the whole energy range, in particular at low energies where multiple scattering leads to generally higher $\chi^{2}$ values. Reconstructed tracks were required to lie entirely inside a fiducial volume with bounding surfaces $0.15 \mathrm{~cm}$ from the magnet walls. Galactic events were selected by imposing that the lower edge of the rigidity bin to which

\footnotetext{
24 The MDR is defined as the rigidity with an associated $100 \%$ error due to the finite spatial resolution of the spectrometer.

$251 \mathrm{MIP}$ is defined here as the most probable energy release of a $|Z|=1$ minimum ionizing particle.
}

the event belongs exceeds the critical rigidity, $\rho_{c}$, defined as 1.3 times the cutoff rigidity $\rho_{\mathrm{SVC}}$ computed in the Störmer vertical approximation (Shea et al. 1987) as $\rho_{\mathrm{SvC}}=14.9 / L^{2}$, where $L$ is the McIlwain $L$-shell parameter (McIlwain 1961) obtained by using the Resurs-DK1 orbital information and the IGRF magnetic field model (MacMillan \& Maus 2005). The South Atlantic Anomaly region has been included in the analysis. Reconstructed particle trajectories were required to be down-going according to the TOF. No selections on the hit pattern in the TOF paddles or AC were made, since this can lead to very low efficiencies due to the production of delta rays in the aluminum dome of the pressurized vessel in which PAMELA is hosted. This introduces a contamination from secondaries produced in hadronic interactions of primaries in the dome. This effect has been accounted for using Monte Carlo simulations.

Boron and carbon events have been selected by means of ionization energy losses in the TOF system. Charge consistency has been required between $\mathrm{S} 12^{26}$ and $\langle\mathrm{S} 2\rangle$ and $\langle\mathrm{S} 3\rangle$ (the arithmetic mean of the ionizations for the two layers constituting $\mathrm{S} 2$ and S3, respectively). Requiring charge consistency above and below the tracking system rejected events interacting in the silicon layers. The selection bands as functions of the rigidity measured by the spectrometer are shown in Figure 2.

In order to assess the presence of possible contamination in the selected samples, the above selection cuts have been applied to boron and carbon samples independently selected by means of S11 (the upper layer of S1) and the first silicon layer of the calorimeter. The probabilities of misidentifying a carbon nucleus as boron and vice versa are about $3 \times 10^{-4}$ and $10^{-3}$, respectively, over the whole energy range considered in this analysis. Stricter analysis criteria were imposed by narrowing the selection bands. When properly corrected by the selection efficiency (see Section 3.3), the event counts showed no statistically significant deviation from that obtained using the standard selection. The contamination is therefore assumed to be negligible. Selected events have been binned according to the rigidity measured by the magnetic spectrometer.

\subsection{Efficiencies}

The tracking efficiency has been evaluated with flight data and Monte Carlo simulations using a methodology similar to that described in (Adriani et al. 2013). Two samples of boron and carbon were selected by means of a $\beta$ dependent requirement on ionization energy losses in the TOF system. Fiducial containment was verified using calorimeter information. Firstly, non-interacting events penetrating deeply into the calorimeter were identified, and a straight track fitted. Then, the rigidity of the nucleus was derived from the $\beta$ measured by the TOF and used to back-propagate the track through the spectrometer magnetic field up to the top of the apparatus. The containment criteria were applied to this back-extrapolated track. The tracking efficiency was determined for this sample of non-interacting nuclei as a function of the rigidity derived from $\beta$. The $70 \mathrm{ps}$ resolution of the TOF system for carbon leads to $\Delta \beta / \beta \sim 2 \%$ at $\beta=0.9$ (Campana et al. 2009). Bin folding effects on the efficiency have therefore been neglected.

Due to the calorimeter selection criteria described above, the efficiency is measured for a non-isotropically distributed sample, while the fluxes impinging on PAMELA are isotropic.

\footnotetext{
$26 \mathrm{~S} 12$ is the lowest of the two layers constituting S1; the upper layer S11 was used for efficiency measurement as explained in Section 3.3.
} 

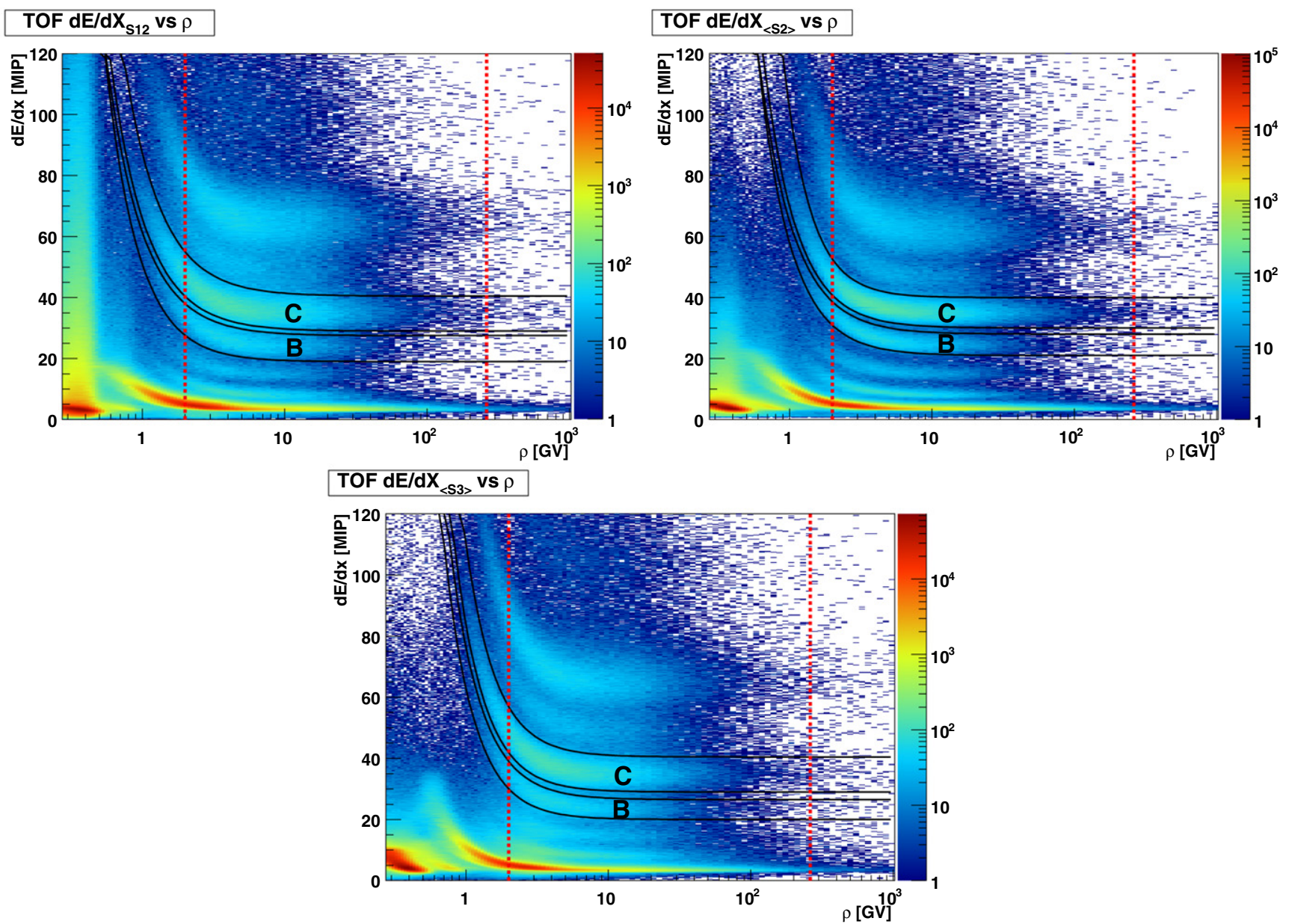

Figure 2. Charge selection bands for $\mathrm{S} 12,\langle\mathrm{~S} 2\rangle$, and $\langle\mathrm{S} 3\rangle$ as a function of rigidity. The red vertical dotted lines denote the upper and lower rigidity limits of this analysis. The absence of relativistic protons in this sample is due to the 5 MIP cluster selection described in Section 3.1.

(A color version of this figure is available in the online journal.)

Moreover, a possible energy dependence of the efficiency at relativistic energies cannot be accounted for by an efficiency measured as a function of $\beta$. To account for these effects, a simulation of the PAMELA apparatus based on GEANT4 (Agostinelli et al. 2003; Allison et al. 2006) has been used to estimate the isotropic, rigidity-dependent tracking efficiency which is subsequently divided by a Monte Carlo efficiency obtained using the same procedure as the experimental efficiency. The resulting ratio, which has an almost constant value of about 0.97 , has been used as the correction factor for the experimental efficiency. The constancy of the ratio results from an isotropic efficiency that is also almost constant above $10 \mathrm{GV}$ because of the data processing procedures described in Section 3.1.

The efficiencies for the selection of down-going particles and for charge selection have been estimated using flight data exclusively. The down-going requirement is $100 \%$ efficient due to the $70 \mathrm{ps}$ resolution of the TOF system. To evaluate the charge selection efficiency, the redundancy of the PAMELA subdetectors has been exploited. Two samples of boron and carbon have been tagged requiring charge consistency on S11 and on the first silicon layer of the calorimeter. These two detectors are placed at the two extrema of the apparatus, so this selection rejects interactions which change the reconstructed charge of the incident particle. The resulting efficiencies have a peak value of $\sim 75 \%$ at $3 \mathrm{GV}$ and then decrease at high energies toward an almost constant value of about $50 \%$ for boron and $60 \%$ for carbon above some tens of GV.
The tracking and the charge selection efficiencies are shown in Figure 3 together with the total selection efficiency.

The measurement of the charge selection efficiency sets the lower rigidity limit for fluxes to $2 \mathrm{GV}$, corresponding to about $0.44 \mathrm{GeV} / \mathrm{n}$ for ${ }^{10} \mathrm{~B}$ and ${ }^{12} \mathrm{C}$. Below this threshold charge confusion in the calorimeter selection becomes too large to be able to reliably tag pure boron and carbon samples for an efficiency measurement.

The effects of a possible contamination in the efficiency samples tagged with S11 and the calorimeter (S11+CALO tag) have been investigated by considering a single TOF layer and measuring the charge selection efficiency both on the event set tagged with S11+CALO and on a purer sample obtained by adding the other TOF planes to the S11+CALO tag. The two efficiencies were found to be consistent within statistical errors for each layer. No effect due to contamination in the S11+CALO tagged set was observed.

\subsection{Corrections}

The selected boron and carbon samples are contaminated by secondaries produced during fragmentation processes occurring in the aluminum dome on top of the pressurized vessel hosting PAMELA. This effect has been studied with a Monte Carlo calculation based on the FLUKA code (Battistoni et al. 2007) by simulating the cosmic spectra for $\mathrm{C}$ and $\mathrm{O}$, which are the main contributors to the contamination. The resulting contamination 

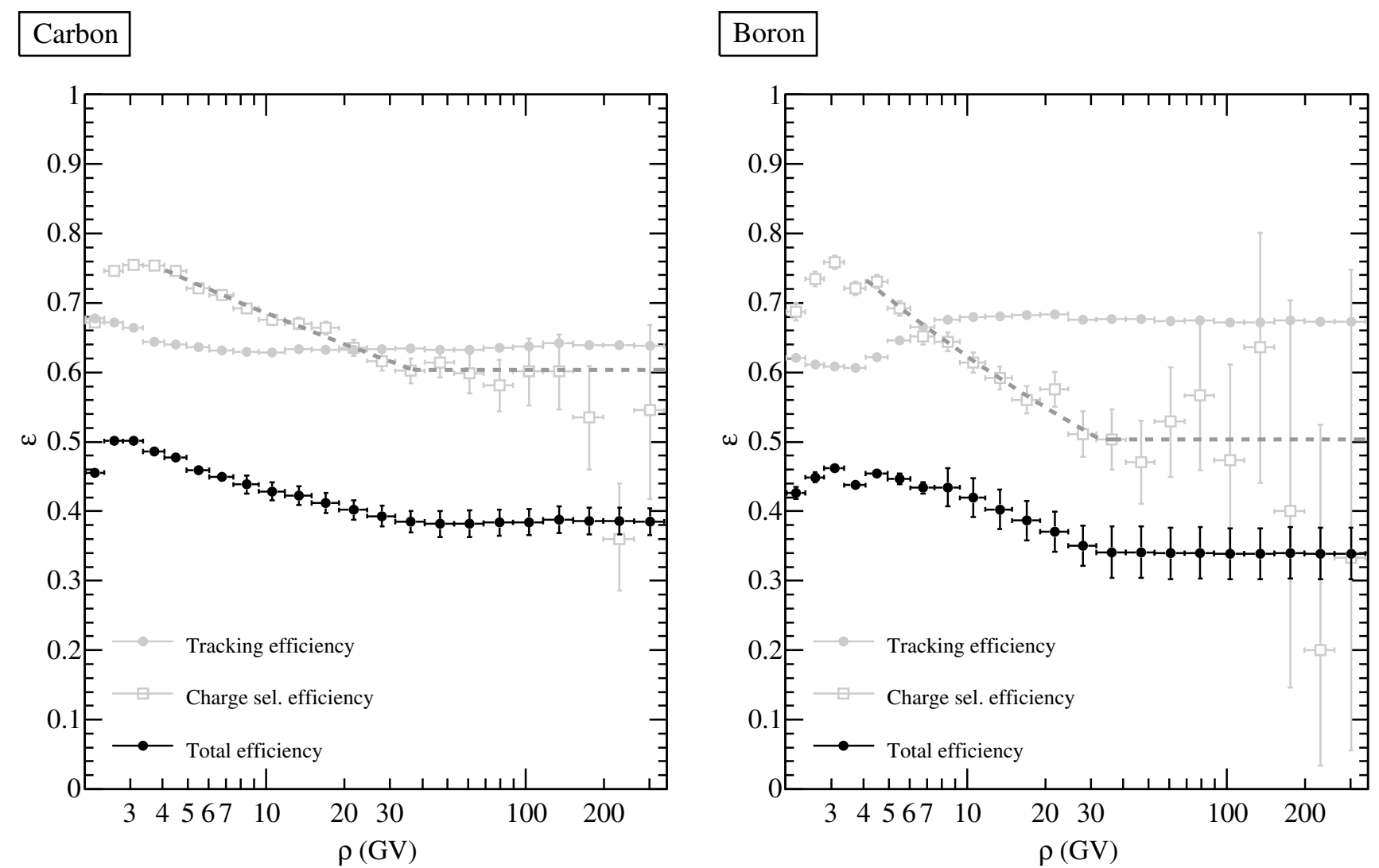

Figure 3. Selection efficiencies as functions of rigidity. The dashed line is a fit of the charge selection efficiency above $3 \mathrm{GV}$ with a power law at low rigidities and a constant value at high rigidities. The slope, the break point and the normalization are free parameters of the fit. The fitted charge selection efficiency is used to compute the total efficiency above $3 \mathrm{GeV} / \mathrm{n}$ (about $7.6 \mathrm{GV}$ for $\mathrm{C}$ and ${ }^{10} \mathrm{~B}$ and $8.4 \mathrm{GV}$ for ${ }^{11} \mathrm{~B}$ ) in order to smooth the statistical fluctuations.

is of the order of $10^{-3}$ for carbon, whereas for boron it ranges from about $5 \%$ at some $\mathrm{GV}$ up to about $20 \%$ at $\sim 200 \mathrm{GV}$, coming mainly from spallation of carbon.

After subtracting the contamination, the rigidity distributions of boron and carbon events have been corrected for folding effects using a Bayesian procedure (D'Agostini 1995), in order to obtain the distributions at the top of payload. These effects include possible rigidity displacements at high energies due to the finite position resolution of the silicon tracking layers and the energy loss of low-energy nuclei traversing the apparatus. The smearing matrix was derived using the GEANT4 simulations.

Interactions with the aluminum dome also remove primaries from the selected samples. Elastic scattering processes can remove primaries from the instrument acceptance or slow them down so that they are swept out by the magnetic field; inelastic scattering can destroy the primary. A correction factor for these effects has been evaluated using the FLUKA simulations, and applied to the unfolded event count. The correction is almost flat above $10 \mathrm{GV}$ and amounts to $15 \%$ for carbon and $14 \%$ for boron, increasing at lower energies because of energy loss. These numbers have been treated as corrections to the geometrical factor for the two nuclear species. The resulting geometrical factors are shown in Figure 4.

Energy loss in the apparatus may lower the measured rigidity below the critical rigidity, leading to rejection of galactic nuclei with initial rigidity above the critical one. A "cutoff correction factor" for each nuclear species was computed by assigning a random cutoff value (distributed as observed for in-flight values) to events simulated with GEANT4 and deriving the fraction of rejected events. This correction factor rises from about 0.97 at $2 \mathrm{GV}$ to unity (i.e., no correction) at $3 \mathrm{GV}$ and above.

\subsection{Live Time}

The live time of the apparatus is measured by on-board clocks and has been evaluated as a function of the vertical cutoff as the time spent in regions where the critical rigidity is below the lower limit of the rigidity bin. The total live time is constant at a value of $\sim 3.14 \times 10^{7} \mathrm{~s}$ for rigidities above $20 \mathrm{GV}$ and decreases at lower rigidities because of the shorter time spent by the satellite in high latitude (i.e., low cutoff) regions down to $\sim 1.00 \times 10^{7} \mathrm{~s}$ at $2 \mathrm{GV}$. The overall error on live time determination is less than $0.2 \%$, and has therefore been neglected.

\subsection{Geometrical Factor}

Due to the requirement of track containment inside a fiducial volume (see Section 3.2), the effective geometrical factor turns out to be lower than the nominal one, and assumes a constant value of $19.9 \mathrm{~cm}^{2}$ sr above $1 \mathrm{GV}$. This value has been crosschecked using two different numerical methods. The first one is a numerical computation of the integral defining the geometrical factor (Sullivan 1971), taking into account the curvature of the track due to the magnetic field, while the second method relies on a Monte Carlo simulation (Sullivan 1971). The two methods yield results differing by less than $0.1 \%$. This error has also been neglected.

\subsection{Flux Computation}

The fluxes have been computed both as functions of rigidity and as functions of kinetic energy per nucleon. For each bin $i$, the event count $\Delta N_{i}^{\prime}$ corrected for the effects described in Section 3.4 was divided by the live time $\Delta T_{i}$, the effective geometrical factor 


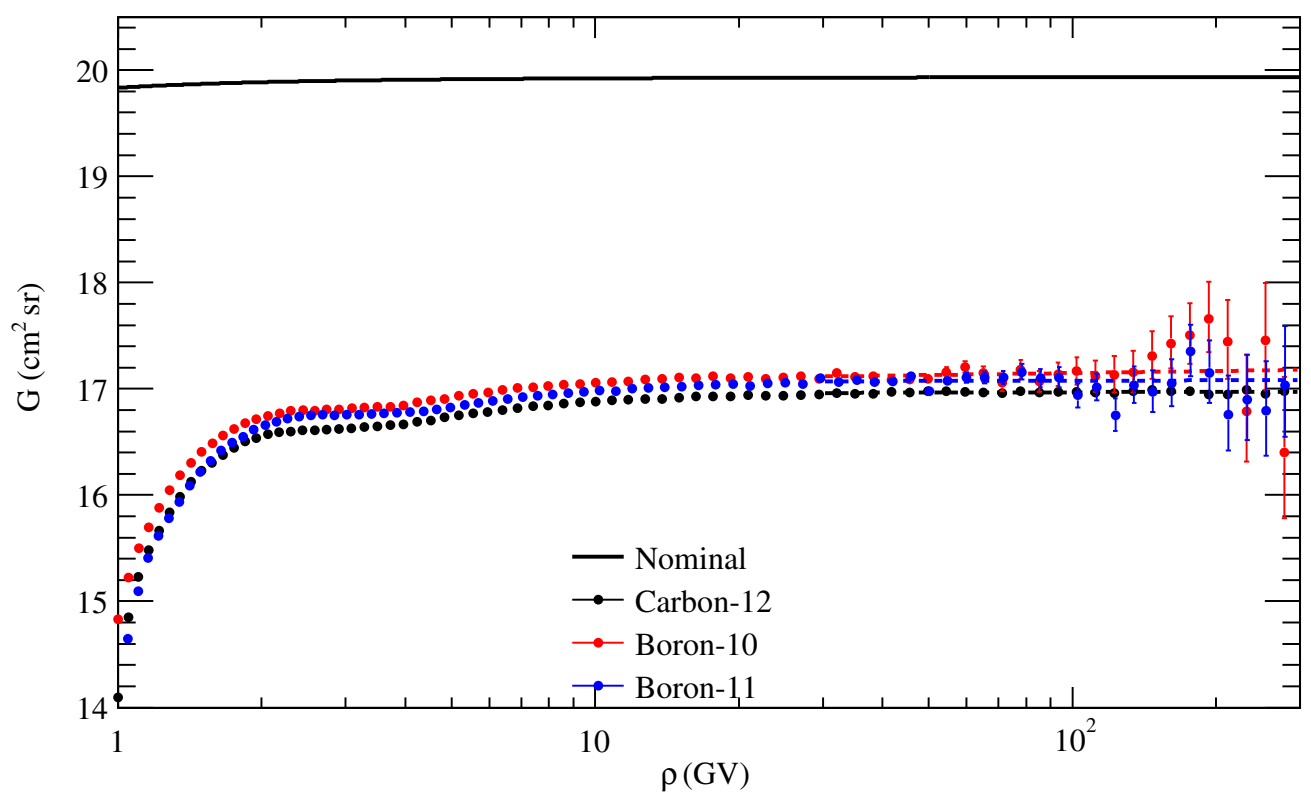

Figure 4. Effective geometrical factors including the fiducial containment criterion and the correction for interactions of primary particles above the tracker. The dashed lines are fits used to obtain asymptotic values at high energy.

(A color version of this figure is available in the online journal.)

$\tilde{G}_{i}$, the total selection efficiency $\epsilon_{i}$ and the bin width $\Delta \rho_{i}$ or $\Delta E_{i}$. The flux expressed as a function of rigidity is computed as

$$
\phi\left(\rho_{i}\right)=\frac{\Delta N_{i}^{\prime}}{\Delta T_{i} \tilde{G}_{i} \epsilon_{i} \Delta \rho_{i}},
$$

while as a function of kinetic energy per nucleon:

$$
\phi\left(E_{i}\right)=\frac{\Delta N_{i}^{\prime}}{\Delta T_{i} \tilde{G}_{i} \epsilon_{i} \Delta E_{i}} .
$$

For boron, the latter formula needs to properly account for isotopic composition, as explained in the next section.

\subsection{Isotopic Composition for Boron}

In cosmic rays, both the isotopes ${ }^{10} \mathrm{~B}$ and ${ }^{11} \mathrm{~B}$ are present in comparable quantities. Since the event selection did not distinguish between them and since the events are binned according to their rigidity, a given value for the isotopic composition of boron must be assumed in order to perform the measurements as functions of kinetic energy per nucleon. Large uncertainties plague the available estimates of the isotopic composition of boron. Direct measurements are available only at relatively low energies (Ahlen et al. 2000; Hams et al. 2004; Aguilar et al. 2011). Galactic propagation models predict a highenergy value for the ${ }^{10} \mathrm{~B}$ fraction (i.e., $\left.{ }^{10} \mathrm{~B} /\left({ }^{10} \mathrm{~B}+{ }^{11} \mathrm{~B}\right)\right)$ which is weakly dependent on kinetic energy per nucleon and whose consensus value is $\tilde{F}_{B}=0.35 \pm 0.15$. This value has been used in this analysis for the whole energy range.

The boron flux has been evaluated considering two different hypotheses: pure ${ }^{10} \mathrm{~B}$ and pure ${ }^{11} \mathrm{~B}$. Assuming a binning in kinetic energy per nucleon, the corresponding binning in rigidity for each of the two hypotheses has been derived. Event selection, efficiency measurements, flux computation and corrections have then been performed in the same way for the two binnings. The two boron fluxes are combined to obtain the final flux, considering that each bin of each flux distribution contains ${ }^{10} \mathrm{~B}$ and ${ }^{11} \mathrm{~B}$ events with the same rigidity but different energy due to the different masses. Consequently, in each bin the isotopic fraction does not resemble the usual fraction expressed as a function of kinetic energy per nucleon, and a simple bin-by-bin linear combination of the two fluxes using $\tilde{F}_{B}$ as the weight would lead to an incorrect result. A fraction $F_{B}(\rho)$ has been derived by means of Monte Carlo simulations and used as a weight in order to linearly combine the two boron fluxes bin by bin and obtain a final flux. A detailed description of the calculation is presented in Appendix B.

\section{RESULTS}

The observed number of selected boron and carbon events, the absolute fluxes and the B/C flux ratio are reported in Tables 1 and 2. The quoted systematic uncertainties are discussed in detail in Appendix A. The fluxes and the B/C ratio are also shown in Figures 5 and 6 along with measurements from other experiments and a theoretical calculation based on GALPROP. The details of the calculation are described in Section 5. The mean kinetic energy $\langle E\rangle$ and the mean rigidity $\langle\rho\rangle$ for each bin have been computed according to (Lafferty \& Wyatt 1995) using an iterative procedure starting from the middle point of each bin. The resulting mean energies and rigidities for boron and carbon differ by less than $1 \%$ and have been considered to be equal.

The discrepancies with other experiments at low energies can be reasonably ascribed to solar modulation effects. The data used for this analysis were taken by PAMELA during an unusually quiet solar minimum period, resulting in an enhanced flux of galactic cosmic rays at low energies in the heliosphere, which has already been observed for protons (Adriani et al. 2013) and nuclei (Mewaldt et al. 2010). Above $6 \mathrm{GeV} / \mathrm{n}$ the fluxes are in overall agreement with the other available measurements, especially with those from HEAO and CREAM. A power-law fit above $20 \mathrm{GeV} / \mathrm{n}$ results in a spectral index $\gamma_{B}=3.01 \pm 0.13$ for boron and $\gamma_{C}=2.72 \pm 0.06$ for carbon.

\section{DISCUSSION}

A comprehensive and detailed study of the results presented above is beyond the scope of this paper. The following 
Table 1

Observed Number of Events, Absolute Fluxes, and the B/C Flux Ratio as Function of Kinetic Energy per Nucleon

\begin{tabular}{|c|c|c|c|c|c|c|c|}
\hline $\begin{array}{l}\text { Kinetic Energy } \\
\text { at Top of Payload } \\
(\mathrm{GeV} / \mathrm{n})\end{array}$ & $\begin{array}{c}\langle E\rangle \\
(\mathrm{GeV} / \mathrm{n})\end{array}$ & C Events & $\begin{array}{c}\text { C Flux } \\
\text { Value } \pm \text { Stat. } \pm \text { Syst. } \\
\left(\mathrm{GeV} / \mathrm{n} \mathrm{m}^{2} \mathrm{~s} \mathrm{sr}\right)^{-1}\end{array}$ & ${ }^{10} \mathrm{~B}$ Events & ${ }^{11}$ B Events & $\begin{array}{c}\text { B Flux } \\
\text { Value } \pm \text { Stat. } \pm \text { Syst. } \\
\left(\mathrm{GeV} / \mathrm{n} \mathrm{m}^{2} \mathrm{~s} \mathrm{sr}\right)^{-1}\end{array}$ & $\begin{array}{c}\text { B/C } \\
\text { Value } \pm \text { Stat. } \pm \text { Syst. }\end{array}$ \\
\hline $0.44-0.58$ & 0.49 & 5146 & $(5.26 \pm 0.08 \pm 0.26)$ & 1566 & 1795 & $\left(1.73 \pm 0.04_{-0.08}^{+0.09}\right)$ & $\left(3.28 \pm 0.09_{-0.22}^{+0.23}\right) \times 10^{-1}$ \\
\hline $0.58-0.76$ & 0.65 & 6651 & $(4.27 \pm 0.05 \pm 0.21)$ & 1955 & 2092 & $\left(1.38 \pm 0.03_{-0.06}^{+0.07}\right)$ & $\left(3.24 \pm 0.07_{-0.21}^{+0.23}\right) \times 10^{-1}$ \\
\hline $0.76-1.00$ & 0.85 & 7359 & $(3.30 \pm 0.04 \pm 0.16)$ & 2300 & 2320 & $\left(1.102 \pm 0.020_{-0.050}^{+0.059}\right)$ & $\left(3.34 \pm 0.07_{-0.22}^{+0.24}\right) \times 10^{-1}$ \\
\hline $1.00-1.30$ & 1.13 & 7578 & $(2.45 \pm 0.03 \pm 0.12)$ & 2351 & 2248 & $\left(7.85 \pm 0.14_{-0.36}^{+0.42}\right) \times 10^{-1}$ & $\left(3.21 \pm 0.07_{-0.21}^{+0.23}\right) \times 10^{-1}$ \\
\hline $1.30-1.71$ & 1.50 & 7033 & $(1.612 \pm 0.019 \pm 0.078)$ & 2281 & 2166 & $\left(5.18 \pm 0.10_{-0.24}^{+0.29}\right) \times 10^{-1}$ & $\left(3.22 \pm 0.07_{-0.22}^{+0.24}\right) \times 10^{-1}$ \\
\hline $1.71-2.24$ & 1.94 & 6369 & $(1.057 \pm 0.013 \pm 0.051)$ & 1960 & 1737 & $\left(3.06 \pm 0.06_{-0.15}^{+0.17}\right) \times 10^{-1}$ & $\left(2.89 \pm 0.07_{-0.20}^{+0.22}\right) \times 10^{-1}$ \\
\hline $2.24-2.93$ & 2.53 & 5673 & $(6.70 \pm 0.09 \pm 0.32) \times 10^{-1}$ & 1691 & 1553 & $\left(1.88 \pm 0.04_{-0.09}^{+0.11}\right) \times 10^{-1}$ & $\left(2.80 \pm 0.07_{-0.20}^{+0.21}\right) \times 10^{-1}$ \\
\hline $2.93-3.84$ & 3.34 & 4795 & $(3.99 \pm 0.06 \pm 0.20) \times 10^{-1}$ & 1350 & 1202 & $(1.03 \pm 0.03 \pm 0.07) \times 10^{-1}$ & $\left(2.59 \pm 0.09_{-0.21}^{+0.23}\right) \times 10^{-1}$ \\
\hline $3.84-5.03$ & 4.36 & 3990 & $(2.32 \pm 0.04 \pm 0.12) \times 10^{-1}$ & 1078 & 945 & $(5.8 \pm 0.2 \pm 0.4) \times 10^{-2}$ & $\left(2.49 \pm 0.10_{-0.21}^{+0.22}\right) \times 10^{-1}$ \\
\hline $5.03-6.60$ & 5.73 & 3270 & $(1.31 \pm 0.02 \pm 0.07) \times 10^{-1}$ & 811 & 704 & $\left(3.05 \pm 0.12_{-0.22}^{+0.23}\right) \times 10^{-2}$ & $\left(2.32 \pm 0.10_{-0.20}^{+0.21}\right) \times 10^{-1}$ \\
\hline $6.60-8.65$ & 7.49 & 2717 & $(7.32 \pm 0.14 \pm 0.38) \times 10^{-2}$ & 612 & 540 & $(1.56 \pm 0.07 \pm 0.12) \times 10^{-2}$ & $\left(2.134 \pm 0.10_{-0.19}^{+0.20}\right) \times 10^{-1}$ \\
\hline $8.65-11.3$ & 9.81 & 2048 & $(3.65 \pm 0.08 \pm 0.19) \times 10^{-2}$ & 454 & 369 & $(7.8 \pm 0.4 \pm 0.6) \times 10^{-3}$ & $(2.128 \pm 0.12 \pm 0.20) \times 10^{-1}$ \\
\hline $11.3-14.9$ & 12.9 & 1337 & $(1.81 \pm 0.05 \pm 0.10) \times 10^{-2}$ & 253 & 217 & $(3.6 \pm 0.2 \pm 0.3) \times 10^{-3}$ & $\left(1.99 \pm 0.13_{-0.19}^{+0.20}\right) \times 10^{-1}$ \\
\hline $14.9-19.5$ & 16.9 & 851 & $(9.0 \pm 0.3 \pm 0.5) \times 10^{-3}$ & 149 & 121 & $\left(1.56 \pm 0.12_{-0.12}^{+0.13}\right) \times 10^{-3}$ & $(1.73 \pm 0.15 \pm 0.17) \times 10^{-1}$ \\
\hline $19.5-25.5$ & 22.1 & 571 & $(4.6 \pm 0.2 \pm 0.3) \times 10^{-3}$ & 85 & 69 & $(6.7 \pm 0.7 \pm 0.6) \times 10^{-4}$ & $(1.45 \pm 0.16 \pm 0.14) \times 10^{-1}$ \\
\hline $25.5-43.8$ & 32.6 & 590 & $(1.67 \pm 0.07 \pm 0.07) \times 10^{-3}$ & 79 & 65 & $(2.1 \pm 0.2 \pm 0.1) \times 10^{-4}$ & $(1.22 \pm 0.13 \pm 0.09) \times 10^{-1}$ \\
\hline $43.8-75.3$ & 55.7 & 225 & $(3.8 \pm 0.3 \pm 0.2) \times 10^{-4}$ & 31 & 24 & $(4.2 \pm 0.6 \pm 0.3) \times 10^{-5}$ & $(1.11 \pm 0.18 \pm 0.08) \times 10^{-1}$ \\
\hline $75.3-129$ & 95.6 & 86 & $(8.5 \pm 0.8 \pm 0.4) \times 10^{-5}$ & 9 & 7 & $(8.4 \pm 1.5 \pm 0.5) \times 10^{-6}$ & $(10 \pm 2 \pm 0.7) \times 10^{-2}$ \\
\hline
\end{tabular}

Note. Both the event counts for pure ${ }^{10} \mathrm{~B}$ and pure ${ }^{11} \mathrm{~B}$ hypotheses are reported.

Table 2

Observed Number of Events, Absolute Fluxes, and the B/C Flux Ratio as Function of Rigidity

\begin{tabular}{|c|c|c|c|c|c|c|}
\hline $\begin{array}{l}\text { Rigidity } \\
\text { at Top of Payload } \\
(\mathrm{GV})\end{array}$ & $\langle\rho\rangle$ & C Events & $\begin{array}{c}\text { C Flux } \\
\text { Value } \pm \text { Stat. } \pm \text { Syst. } \\
\left(\mathrm{GV} \mathrm{m} \mathrm{m}^{2} \mathrm{~s} \mathrm{sr}\right)^{-1}\end{array}$ & B Events & $\begin{array}{c}\text { B Flux } \\
\text { Value } \pm \text { Stat. } \pm \text { Syst. } \\
\left(\mathrm{GV} \mathrm{m} \mathrm{m}^{2} \mathrm{~s} \mathrm{sr}\right)^{-1}\end{array}$ & $\begin{array}{c}\text { B/C } \\
\text { Value } \pm \text { Stat. } \pm \text { Syst. }\end{array}$ \\
\hline $2.02-2.38$ & 2.19 & 5146 & $(2.01 \pm 0.03 \pm 0.10)$ & 1566 & $(6.26 \pm 0.16 \pm 0.29) \times 10^{-1}$ & $(3.12 \pm 0.09 \pm 0.21) \times 10^{-1}$ \\
\hline $2.38-2.82$ & 2.57 & 6651 & $(1.73 \pm 0.02 \pm 0.08)$ & 1955 & $(5.49 \pm 0.13 \pm 0.25) \times 10^{-1}$ & $(3.17 \pm 0.08 \pm 0.21) \times 10^{-1}$ \\
\hline $2.82-3.37$ & 3.06 & 7359 & $(1.413 \pm 0.017 \pm 0.068)$ & 2300 & $(4.72 \pm 0.10 \pm 0.21) \times 10^{-1}$ & $(3.34 \pm 0.08 \pm 0.22) \times 10^{-1}$ \\
\hline $3.37-4.06$ & 3.67 & 7578 & $(1.093 \pm 0.013 \pm 0.053)$ & 2351 & $(3.67 \pm 0.08 \pm 0.17) \times 10^{-1}$ & $(3.35 \pm 0.08 \pm 0.22) \times 10^{-1}$ \\
\hline $4.06-4.93$ & 4.45 & 7033 & $(7.44 \pm 0.09 \pm 0.36) \times 10^{-1}$ & 2281 & $(2.52 \pm 0.05 \pm 0.11) \times 10^{-1}$ & $(3.39 \pm 0.08 \pm 0.22) \times 10^{-1}$ \\
\hline $4.93-6.06$ & 5.44 & 6369 & $(5.00 \pm 0.06 \pm 0.24) \times 10^{-1}$ & 1960 & $(1.56 \pm 0.04 \pm 0.07) \times 10^{-1}$ & $(3.12 \pm 0.08 \pm 0.21) \times 10^{-1}$ \\
\hline $6.06-7.50$ & 6.70 & 5673 & $(3.23 \pm 0.04 \pm 0.16) \times 10^{-1}$ & 1691 & $(9.9 \pm 0.2 \pm 0.5) \times 10^{-2}$ & $(3.06 \pm 0.08 \pm 0.21) \times 10^{-1}$ \\
\hline $7.50-9.36$ & 8.34 & 4795 & $(1.95 \pm 0.03 \pm 0.10) \times 10^{-1}$ & 1350 & $(5.52 \pm 0.15 \pm 0.34) \times 10^{-2}$ & $(2.83 \pm 0.09 \pm 0.23) \times 10^{-1}$ \\
\hline $9.36-11.8$ & 10.4 & 3990 & $(1.143 \pm 0.018 \pm 0.058) \times 10^{-1}$ & 1078 & $(3.19 \pm 0.10 \pm 0.20) \times 10^{-2}$ & $(2.79 \pm 0.10 \pm 0.23) \times 10^{-1}$ \\
\hline $11.8-15.0$ & 13.2 & 3270 & $(6.49 \pm 0.11 \pm 0.33) \times 10^{-2}$ & 811 & $(1.70 \pm 0.06 \pm 0.11) \times 10^{-2}$ & $(2.61 \pm 0.10 \pm 0.22) \times 10^{-1}$ \\
\hline $15.0-19.1$ & 16.8 & 2717 & $(3.64 \pm 0.07 \pm 0.19) \times 10^{-2}$ & 612 & $(8.8 \pm 0.4 \pm 0.6) \times 10^{-3}$ & $(2.43 \pm 0.11 \pm 0.21) \times 10^{-1}$ \\
\hline $19.1-24.5$ & 21.4 & 2048 & $(1.82 \pm 0.04 \pm 0.10) \times 10^{-2}$ & 454 & $(4.4 \pm 0.2 \pm 0.3) \times 10^{-3}$ & $(2.42 \pm 0.13 \pm 0.21) \times 10^{-1}$ \\
\hline $24.5-31.5$ & 27.6 & 1337 & $(9.1 \pm 0.3 \pm 0.5) \times 10^{-3}$ & 253 & $(2.02 \pm 0.13 \pm 0.15) \times 10^{-3}$ & $(2.24 \pm 0.15 \pm 0.20) \times 10^{-1}$ \\
\hline $31.5-40.8$ & 35.6 & 851 & $(4.51 \pm 0.16 \pm 0.24) \times 10^{-3}$ & 149 & $(8.9 \pm 0.7 \pm 0.7) \times 10^{-4}$ & $(1.96 \pm 0.18 \pm 0.18) \times 10^{-1}$ \\
\hline $40.8-52.9$ & 46.1 & 571 & $(2.32 \pm 0.10 \pm 0.13) \times 10^{-3}$ & 85 & $(3.9 \pm 0.4 \pm 0.3) \times 10^{-4}$ & $(1.7 \pm 0.2 \pm 0.16) \times 10^{-1}$ \\
\hline $52.9-89.5$ & 67.1 & 590 & $(8.4 \pm 0.4 \pm 0.3) \times 10^{-4}$ & 79 & $(1.18 \pm 0.14 \pm 0.07) \times 10^{-4}$ & $(1.41 \pm 0.18 \pm 0.10) \times 10^{-1}$ \\
\hline $89.5-152$ & 113 & 225 & $(1.92 \pm 0.14 \pm 0.08) \times 10^{-4}$ & 31 & $(2.5 \pm 0.5 \pm 0.1) \times 10^{-5}$ & $(1.3 \pm 0.3 \pm 0.09) \times 10^{-1}$ \\
\hline $152-260$ & 193 & 86 & $(4.3 \pm 0.4 \pm 0.2) \times 10^{-5}$ & 9 & $(4.7 \pm 1.1 \pm 0.3) \times 10^{-6}$ & $(1.1 \pm 0.3 \pm 0.08) \times 10^{-1}$ \\
\hline
\end{tabular}

discussion is intentionally limited to a single propagation model in order to compute an estimate of the most significant propagation parameters from the PAMELA boron and carbon data. Results may vary when considering different models or propagation software packages.

The data presented in the previous section as a function of kinetic energy per nucleon has been fitted with a diffusive cosmic ray propagation model using the GALPROP code interfaced with the MIGRAD minimizer in the MINUIT2 minimization package distributed within the ROOT framework (Brun \& Rademakers 1997). Only a few parameters have been left free because of the high computation time required for multiple
GALPROP runs. The values for the other parameters have been taken from (Vladimirov 2012). The diffusion coefficient is found to have a fitted slope value of $\delta=0.397 \pm 0.007$ and a normalization factor $D_{0}=(4.12 \pm 0.04) \times 10^{28} \mathrm{~cm}^{2} \mathrm{~s}^{-1}$. Other fitted parameters are the solar modulation parameter in the force-field approximation $\Phi=(0.40 \pm 0.01) \mathrm{GV}$ and the overall normalization of the fluxes $N=1.04 \pm 0.03$. The result of the fit is shown in Figures 5 and 6. A contour plot of the confidence intervals for $\delta$ and $D_{0}$ is shown in Figure 7.

The fitted value for $\delta$ falls between the predicted values for Kolmogorov $(\delta=1 / 3)$ and Kraichnan $(\delta=1 / 2)$ diffusion 

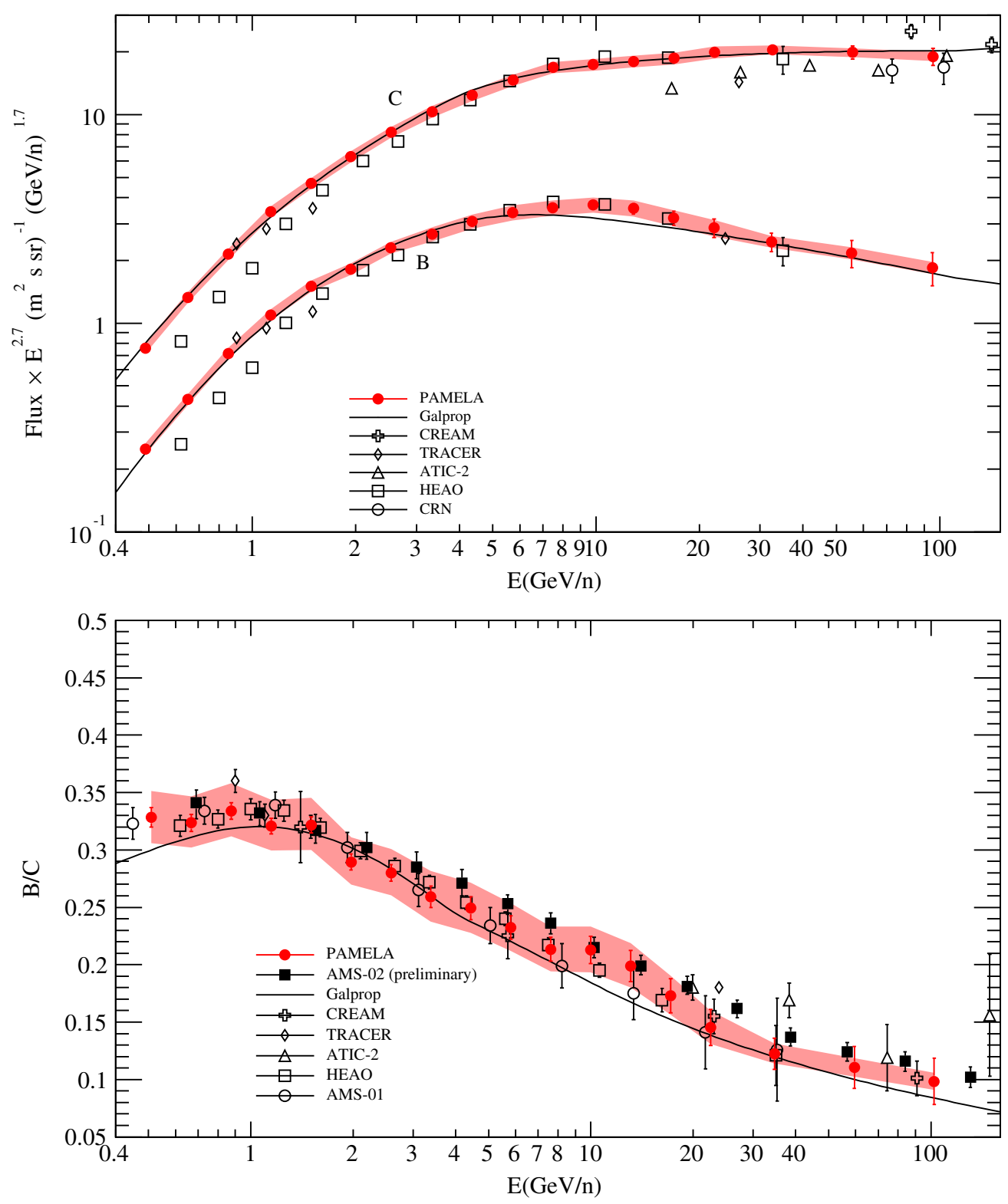

Figure 5. Absolute boron and carbon fluxes multiplied by $E^{2.7}$ (upper panel) and B/C flux ratio (lower panel) as measured by PAMELA, together with results from other experiments (AMS02 (Oliva et al. 2013), CREAM (Ahn et al. 2008), TRACER (Obermeier et al. 2011), ATIC-2 (Panov et al. 2008), HEAO (Engelmann et al. 1990), AMS01 (Aguilar et al. 2010), CRN (Swordy et al. 1990)) and a theoretical calculation based on GALPROP (see Section 5), as functions of kinetic energy per nucleon. For PAMELA data the error bars represent the statistical error and the shaded area is the overall systematic uncertainty summarized in Appendix A.

(A color version of this figure is available in the online journal.)

types, thus the PAMELA data cannot distinguish between these two types.

We acknowledge support from The Italian Space Agency (ASI), Deutsches Zentrum für Luft-und Raumfahrt (DLR), The Swedish National Space Board, The Swedish Research Council, The Russian Space Agency (Roscosmos), and The Russian Science Foundation.

\section{APPENDIX A}

\section{SYSTEMATIC UNCERTAINTIES}

The following contributions to the systematic uncertainty have been considered.

1. Selection efficiencies. The measurement of the tracking and charge selection efficiencies from flight data is performed using samples of finite size. The associated statistical error has been propagated to the flux as a systematic uncertainty.

2. Fiducial containment. The finite tracking resolution of the calorimeter can lead to a contamination of the tracking efficiency sample by events coming from outside the fiducial acceptance, and possibly also crossing the magnet walls. These can in principle be eliminated by further restricting the fiducial volume for both event selection and efficiency measurement, but this would significantly reduce the sample sizes. The chosen approach is to use protons from both flight and simulated data to measure the tracking efficiency for both the fiducial volume defined in Section 3.2 and a more restrictive one. Their relative difference is taken as an estimate of the systematic uncertainty, which is about $2 \%$. Monte Carlo simulations give results for boron and carbon which are consistent with the one obtained with protons. The uncertainty is propagated to the final flux. 

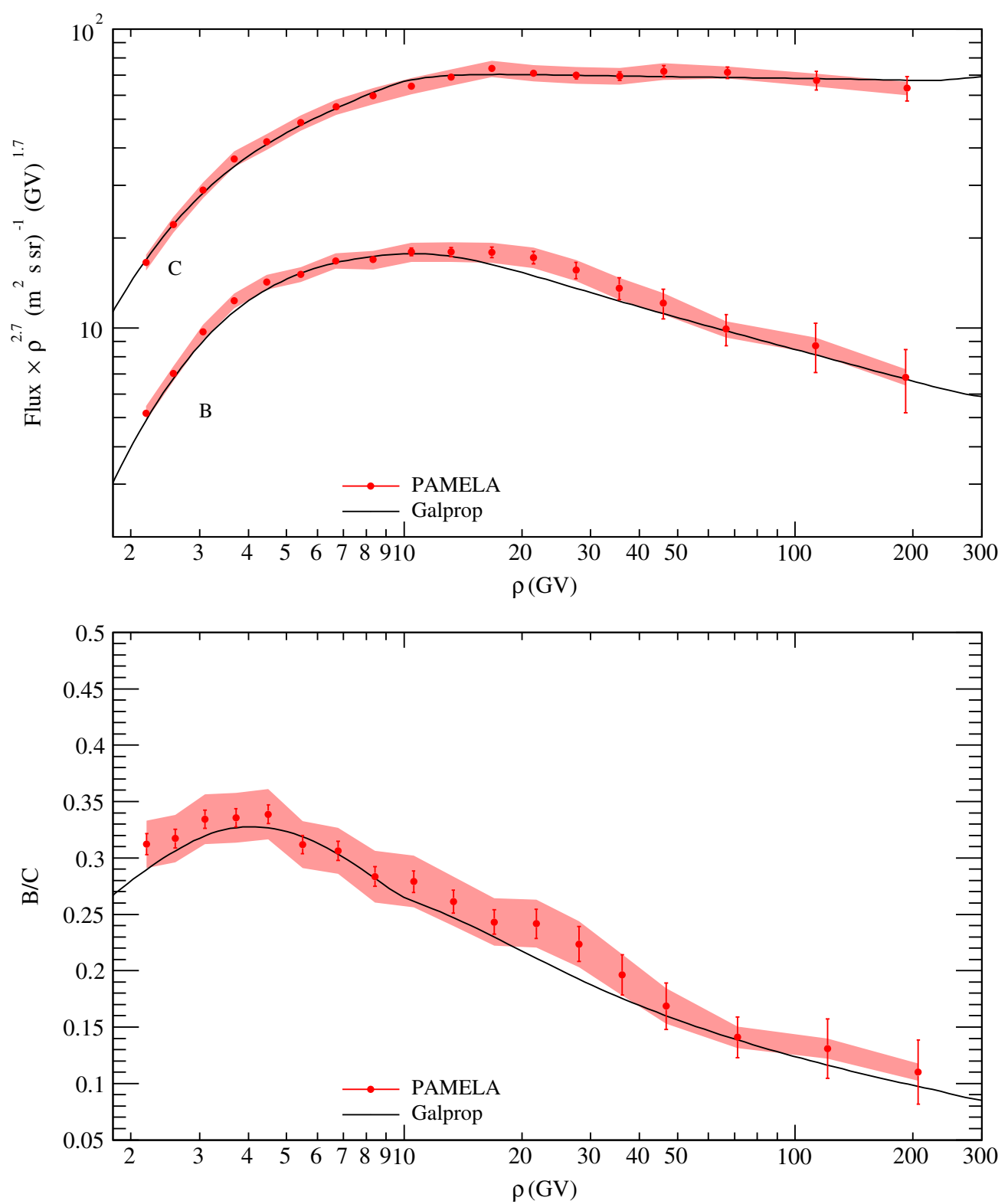

Figure 6. Absolute boron and carbon fluxes multiplied by $\rho^{2.7}$ (upper panel) and B/C flux ratio (lower panel) as measured by PAMELA, together with a theoretical calculation based on GALPROP (see Section 5), as functions of rigidity. The error bars represent the statistical error and the shaded area is the overall systematic uncertainty summarized in Appendix A, except for the boron mixing error which does not affect the rigidity-dependent boron flux.

(A color version of this figure is available in the online journal.)

3. Monte Carlo correction factor for the tracking efficiency. This correction factor should introduce only relatively small errors, since it is computed as the ratio of two Monte Carlo efficiencies. Systematic effects should largely cancel out. The correction factor is constant at 0.97 for both boron and carbon. That this factor remains constant at high rigidity is due to the isotropic efficiency being constant at relativistic rigidities. A conservative factor of 3\% has been taken as an estimate of the systematic uncertainty on the flux because of this correction factor.

4. Residual coherent misalignment of the spectrometer. The spectrometer alignment procedure results in a residual coherent misalignment producing a systematic shift in the measured rigidity. The error estimation procedure is described in the Supporting Online Material of Adriani et al. (2011). This error has been propagated to the measured flux.
It is negligible at low energy and increases up to about $2 \%$ at $250 \mathrm{GV}$.

5. Cutoff, contamination and geometrical factor corrections. All these factors have been evaluated on finite-size samples, so they are affected by a statistical error which has been propagated to the flux as a systematic uncertainty.

6. Unfolding. The unfolding error has been assessed by means of the procedure described in the Supporting Online Material of (Adriani et al. 2011), comparing a given initial spectrum and an unfolded Monte Carlo simulation. The two were found to be in agreement within $3 \%$, so this value has been taken as the unfolding contribution to the flux error.

7. Isotopic composition of boron. The uncertainty associated with this poorly known parameter has been propagated to the flux by assuming the extreme values of 0.2 and 0.5 for the ${ }^{10} \mathrm{~B}$ fraction and taking the difference between these 


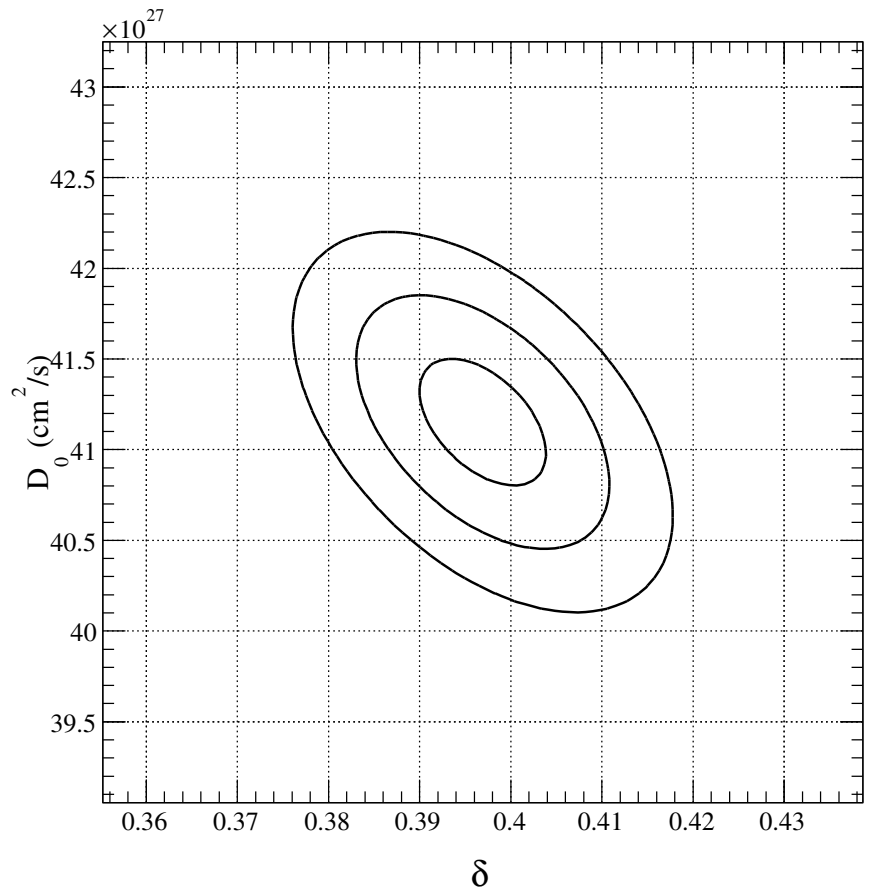

Figure 7. Contour plot of the $1 \sigma, 2 \sigma$ and $3 \sigma$ confidence levels for $\delta$ and $D_{0}$.

fluxes and the one obtained with $\tilde{F}_{B}=0.35$ as the estimated upper and lower errors on the flux. This error affects only the measurement expressed as a function of kinetic energy per nucleon.

The overall uncertainty has been estimated as the quadratic sum of the above terms in the hypothesis of uncorrelated errors. A summary plot is shown in Figure 8.

\section{APPENDIX B}

\section{ISOTOPIC COMPOSITION OF BORON}

In this analysis the events have been binned according to their rigidity as measured by the magnetic spectrometer. Given that the event selection does not distinguish between the two isotopes ${ }^{10} \mathrm{~B}$ and ${ }^{11} \mathrm{~B}$, each bin is populated by ${ }^{10} \mathrm{~B}$ and ${ }^{11} \mathrm{~B}$ events with approximately the same rigidity (within the bin limits) but different kinetic energy per nucleon because of the different mass numbers. Consequently, the isotopic composition in a given bin is not described by the ${ }^{10} \mathrm{~B}$ fraction $F_{B}$ expressed as a function of kinetic energy per nucleon $E$ :

$$
F_{B}(E)=\frac{\phi^{10} B(E)}{\phi^{10} B(E)+\phi^{11} B(E)},
$$

where $\phi^{10} B(E)$ and $\phi^{11} B(E)$ are the fluxes of ${ }^{10} \mathrm{~B}$ and ${ }^{11} \mathrm{~B}$ respectively. A fraction expressed as a function of rigidity must then be derived in order to correctly account for the isotopic composition in each bin:

$$
F_{B}(\rho)=\frac{\phi^{10} B(\rho)}{\phi^{10} B(\rho)+\phi^{11} B(\rho)} .
$$

Using rigidity bins of finite size leads to

$$
F_{B}\left(\rho_{i}\right)=\frac{\Delta N^{10} B\left(\rho_{i}\right)}{\Delta N^{10} B\left(\rho_{i}\right)+\Delta N^{11} B\left(\rho_{i}\right)},
$$

where $F_{B}\left(\rho_{i}\right)$ is the ${ }^{10} \mathrm{~B}$ fraction for the $i$ th rigidity bin centered at $\rho_{i}$, while $\Delta N^{10} B\left(\rho_{i}\right)$ and $\Delta N^{11} B\left(\rho_{i}\right)$ are the ${ }^{10} \mathrm{~B}$ and ${ }^{11} \mathrm{~B}$ event count for the same bin, respectively. $\Delta N^{11} B\left(\rho_{i}\right)$ can be rewritten using the ${ }^{10} \mathrm{~B}$ fraction in kinetic energy:

$$
\Delta N^{11} B\left(\rho_{i}\right)=\Delta N^{11} B\left(E_{i}^{11}\right)=\frac{1-F_{B}\left(E_{i}^{11}\right)}{F_{B}\left(E_{i}^{11}\right)} \Delta N^{10} B\left(E_{i}^{11}\right) .
$$

Here $\Delta N^{11} B\left(E_{i}^{11}\right)$ denotes the ${ }^{11} \mathrm{~B}$ event count in a bin in kinetic energy per nucleon whose limits are obtained by converting the limits in rigidity of the $i$ th bin to kinetic energy assuming the mass and the charge of ${ }^{11} \mathrm{~B} . E_{i}^{11}$ is the kinetic energy per nucleon of a ${ }^{11} \mathrm{~B}$ nucleus of rigidity $\rho_{i}$. Then, by construction, the first equality in the above equation follows. The second equality follows from the definition of $F_{B}\left(E_{i}\right)$ which is the equivalent of Equation (B3) for kinetic energy bins. Note that

$$
\Delta N^{10} B\left(\rho_{i}\right) \neq \Delta N^{10} B\left(E_{i}^{11}\right),
$$

since the limits of the energy and rigidity bins do not correspond for ${ }^{10} \mathrm{~B}$. Converting the bin limits in energy back to rigidity but assuming now the mass and the charge of ${ }^{10} \mathrm{~B}$ yields

$$
\Delta N^{10} B\left(E_{i}^{11}\right)=\Delta N^{10} B\left(\rho_{i}^{\prime}\right) .
$$

$\rho_{i}^{\prime}$ is then the rigidity of a ${ }^{10} \mathrm{~B}$ nucleus having the same kinetic energy per nucleon $E_{i}^{11}$ of a ${ }^{11} \mathrm{~B}$ nucleus of rigidity $\rho_{i}$ (the same relation holds between the limits of the bins centered in $\rho_{i}^{\prime}$ and $\left.\rho_{i}\right)$. To obtain the explicit relationship between $\rho_{i}^{\prime}$ and $\rho$, write $E_{i}^{11}$ as

$$
\rho_{i}^{\prime}=\frac{A_{10}}{Z} \sqrt{\left(E_{i}^{11}\right)^{2}+2 m_{p} E_{i}^{11}},
$$

where $Z$ is the atomic number of boron, $A_{10}$ is the mass number of ${ }^{10} \mathrm{~B}$ and $m_{p}$ is the proton mass, and then $E_{i}^{11}$ as a function of $\rho_{i}$ :

$$
E_{i}^{11}=\sqrt{\frac{Z^{2}}{A_{11}^{2}} \rho_{i}^{2}+m_{p}^{2}}-m_{p},
$$

with $A_{11}$ the mass number of ${ }^{11} \mathrm{~B}$. It follows that

$$
\rho_{i}^{\prime}=\frac{A_{10}}{A_{11}} \rho_{i} .
$$

The final form of the rigidity-dependent ${ }^{10} \mathrm{~B}$ fraction is then

$$
F_{B}\left(\rho_{i}\right) \approx \frac{\Delta N^{10} B\left(\rho_{i}\right)}{\Delta N^{10} B\left(\rho_{i}\right)+\frac{1-\tilde{F}_{B}}{\tilde{F}_{B}} \Delta N^{10} B\left(\rho_{i}^{\prime}\right)},
$$

where the approximated energy-independent value $F_{B}(E) \approx \tilde{F}_{B}$ has been used.

Generally speaking, the fraction expressed as a function of rigidity is not constant and depends on the spectral shape. To account for this a toy Monte Carlo simulation of realistic ${ }^{10} \mathrm{~B}$ and ${ }^{11} \mathrm{~B}$ spectra taken from a galactic propagation model has been set up, the resulting event counts have been trimmed to reproduce $\tilde{F}_{B}=0.35$ and finally the events have been binned according to their rigidity for both the pure ${ }^{10} \mathrm{~B}$ and pure ${ }^{11} \mathrm{~B}$ hypotheses. Knowing the fraction in each rigidity bin of the two binnings one can express the final boron flux in the $i$ th energy bin as

$$
\phi^{B}\left(E_{i}\right)=F_{B_{i}}^{10} \phi_{10}\left(E_{i}\right)+\left(1-F_{B i}^{11}\right) \phi_{11}\left(E_{i}\right),
$$



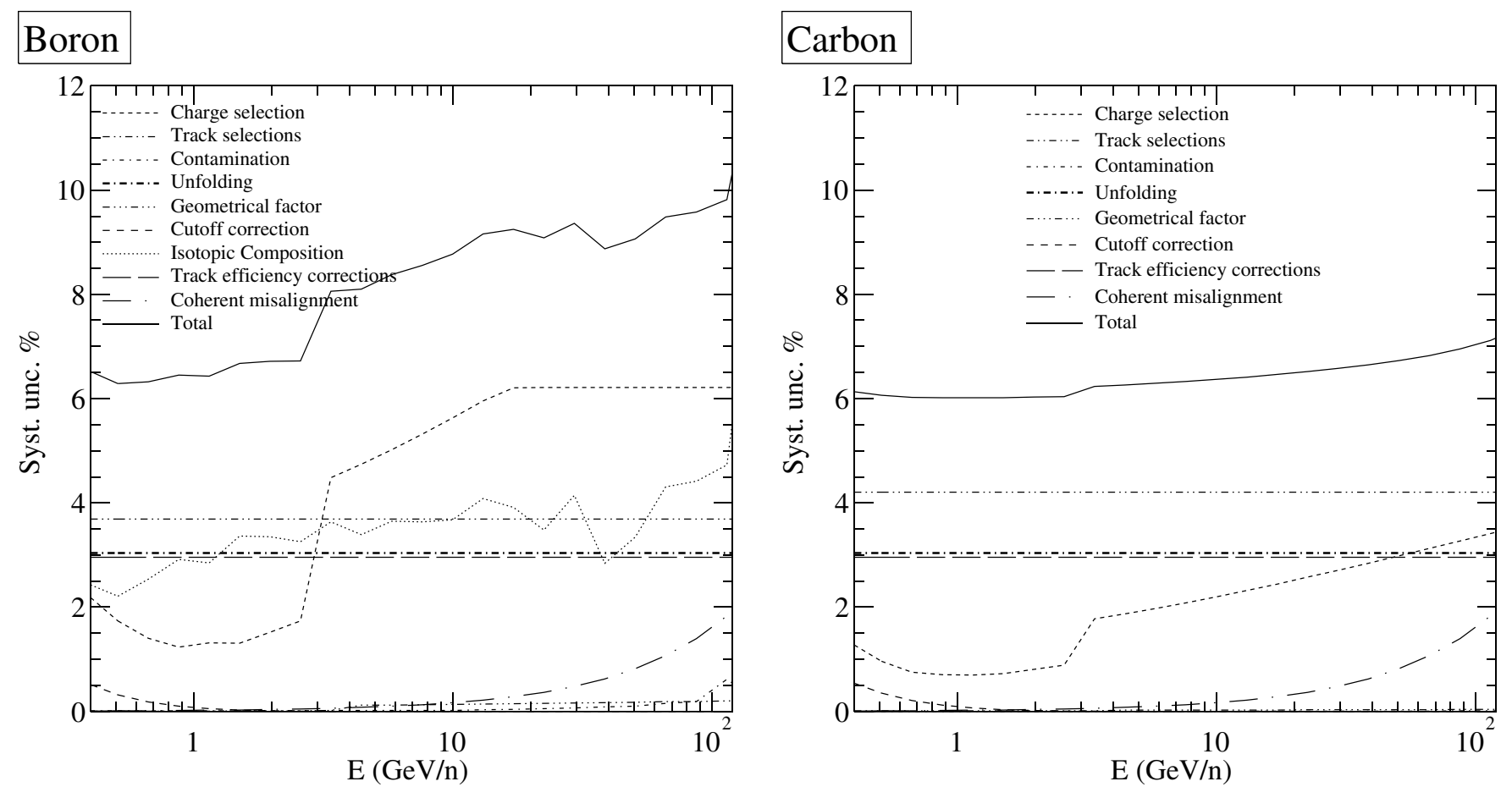

Figure 8. Systematic uncertainties for absolute fluxes. The total contribution is computed as the quadratic sum of the individual terms. The track selections term is the quadratic sum of the contributions from statistics and from fiducial containment. The contributions of the track efficiency correction and of the unfolding have been slightly shifted apart from their $3 \%$ value for the sake of readability.

where $F_{B i}^{10}$ and $F_{B i}^{11}$ are the ${ }^{10} \mathrm{~B}$ fraction obtained from the toy Monte Carlo in the $i$ th rigidity bin for pure ${ }^{10} \mathrm{~B}$ and pure ${ }^{11} \mathrm{~B}$ hypotheses respectively, and $\phi_{10}\left(E_{i}\right)$ and $\phi_{11}\left(E_{i}\right)$ are the experimental fluxes for the pure ${ }^{10} \mathrm{~B}$ and pure ${ }^{11} \mathrm{~B}$ hypotheses respectively (see Section 3.8).

To assess the difference between the ${ }^{10} \mathrm{~B}$ fraction as a function of kinetic energy per nucleon and as a function of rigidity, Equation (B10) can be computed at high energies. Above few $\mathrm{GeV} / \mathrm{n}$, where the spectrum can be well described with a power-law function with index $\gamma$, Equation (B10) gives a ${ }^{10} \mathrm{~B}$ fraction

$$
F_{B}\left(\rho_{i}\right) \approx \frac{1}{1+\frac{1-\tilde{F}_{B}}{\tilde{F}_{B}}\left(A_{10} / A_{11}\right)^{-\gamma}} \simeq 0.288,
$$

which is in agreement with the value obtained from the toy Monte Carlo and differs from $\tilde{F}_{B}=0.35$ by about $18 \%$.

\section{REFERENCES}

Adriani, O., Barbarino, G. C., Bazilevskaya, G. A., et al. 2011, Sci, 332, 69 Adriani, O., Barbarino, G. C., Bazilevskaya, G. A., et al. 2013, ApJ, 765, 91 Adriani, O., Bongi, M., Bonechi, L., et al. 2007, in VERTEX2007, 16th Int. Workshop on Vertex Detectors, ed. M. Artuso, E. Docutoesilva et al. (Trieste, Italy: PoS), 048

Agostinelli, S., Allison, J., Amako, K., et al. 2003, NIMPA, 506, 250

Aguilar, M., Alcaraz, J., Allaby, J., et al. 2010, ApJ, 724, 329

Aguilar, M., Alcaraz, J., Allaby, J., et al. 2011, ApJ, 736, 105

Ahlen, S. P., Greene, N. R., Loomba, D., et al. 2000, ApJ, 534, 757

Ahn, H. S., Allison, P. S., Bagliesi, M. G., et al. 2008, APh, 30, 133

Allison, J., Amako, K., Apostolakis, J., et al. 2006, ITNS, 53, 270

Barbarino, G. C., Campana, D., De Rosa, G., et al. 2008, NIMPA, 584, 319

Battistoni, G., Muraro, S., Sala, P. R., et al. 2007, in Hadronic Simulation

Workshop 2006, ed. M. Albrow \& R. Raja (Melville, NY: AIP), 31

Bethe, H. A. 1939, PhRv, 55, 434
Boezio, M., Bonvicini, V., Mocchiutti, E., et al. 2002, NIMPA, 487, 407 Bonvicini, V., Boezio, M., Mocchiutti, E., et al. 2009, JPhCS, 160, 012039 Brun, R., \& Rademakers, F. 1997, NIMPA, 389, 81

Campana, D., Carbone, R., De Rosa, G., et al. 2009, NIMPA, 598, 696

Castellina, A., \& Donato, F. 2005, APh, 24, 146

D'Agostini, G. 1995, NIMPA, 362, 487

de Nolfo, G. A., Moskalenko, I. V., Binns, W. R., et al. 2006, AdSpR, 38,1558

Engelmann, J. J., Ferrando, P., Soutoul, A., et al. 1990, A\&A, 233, 96

Freier, P. S., Ney, E. P., \& Waddington, C. J. 1959, PhRv, 113, 921

Gaggero, D., Maccione, L., Di Bernardo, G., et al. 2013, PhRvL, 111, 1102

Hams, T., Barbier, L. M., Bremerich, M., et al. 2004, ApJ, 611, 892

Lafferty, G. D., \& Wyatt, T. R. 1995, NIMPA, 355, 541

Lave, K. A., Wiedenbeck, M. E., Binns, W. R., et al. 2013, ApJ, 770, 117

Letaw, J. R., Silberberg, R., \& Tsao, C. H. 1993, ApJ, 414, 601

MacMillan, S., \& Maus, S. 2005, EP\&S, 57, 1135

Maurin, D., Putze, A., \& Derome, L. 2010, A\&A, 516, A67

McIlwain, C. E. 1961, JGR, 66, 3681

Mewaldt, R. A., Davis, A. J., Lave, K. A., et al. 2010, ApJL, 723, L1

Obermeier, A., Ave, M., Boyle, P., et al. 2011, ApJ, 742, 14

Obermeier, A., Boyle, P., Hörandel, J., et al. 2012, ApJ, 752, 69

Oliva, A. 2013, 33rd International Cosmic Ray Conference (ICRC 2013), (available at http://143.107.180.38/indico/contributionListDisplay.py?confid=0)

Osteria, G., \& Russo, S. 2008, NIMPA, 589, 465

Panov, D., Sokolskaya, N. V., Adams, J. H., Jr., et al. 2008, in 30th International Cosmic Ray Conference (ICRC 2007), ed. R. Caballero, J. C. D’Olivo et al. (Mexico City, Mexico: Universidad Nacional Autónoma de México), 3

Pearce, M., Carlson, P., Lund, J., et al. 2003, in 28th International Cosmic Ray Conference (ICRC 2003), ed. T. Kajita, Y. Asaoka et al. (Tokyo, Japan: Univ. Acad. Pr.), 2125

Picozza, P., Galper, A. M., Castellini, G., et al. 2007, APh, 27, 296

Ptuskin, V. 2012, APh, 39, 44

Shea, M. A., Smart, D. F., \& Gentile, L. C. 1987, PEPI, 48, 200

Strong, A., \& Moskalenko, I. V. 1998, ApJ, 509, 212

Strong, A. W., Moskalenko, I. V., \& Ptuskin, V. S. 2007, ARNPS, 57, 285

Sullivan, J. D. 1971, NucIM, 95, 5

Swordy, S. P., Müller, D., Meyer, P., et al. 1990, ApJ, 349, 625

Vladimirov, A. E., Jóhannesson, G., Moskalenko, I. V., \& Porter, T. A. 2012, ApJ, 752, 68

Webber, W. R., Lukasiak, A., \& McDonald, F. B. 2002, ApJ, 568, 210 